\title{
A theory of dropwise condensation at large subcooling including the effect of the sweeping
}

\author{
C. Yamali, H. Merte Jr
}

Abstract The effect of sweeping by the departing droplets on the heat transfer coefficient in dropwise condensation is studied analytically here. Using basic principles, an analytical model for dropwise condensation is devised, which takes into account the elementary processes that make up the dropwise condensation cycle. The analysis is divided into two parts: in the first part, the heat transfer as a result of nucleation and coalescing of the droplets is considered. In the second part, the effect of sweeping is introduced. The results are presented as the variation of nondimensional heat flux versus the distance from the upper edge of the condenser surface at various surface subcoolings. Calculations show that the variation of heat flux with surface subcooling is linear only at small values of subcooling. As the subcooling is increased the slope of the mean heat flux versus subcooling curve decreases, and for a sufficiently high body force passes through a maximum.

\section{List of symbols}

a acceleration

$a_{r} \quad$ component of the acceleration parallel to the condenser surface

A area

$A_{\mathrm{c}} \quad$ condenser surface area

$B \quad$ variable defined by Eq. (7)

$d \quad$ drop base diameter

$D_{\mathrm{d}} \quad$ number of falling droplets per unit area

$D_{\text {dep }}$ base diameter of a departing droplet

$f_{\text {co }}$ fractional area occupied by droplets in the coalescence region

$f_{\mathrm{d}} \quad$ departing frequency of the droplets

$f_{\mathrm{dc}} \quad$ fractional area occupied by primary droplets

$f_{\mathrm{s}} \quad$ sweeping frequency of the droplets

$g$ gravitational acceleration

$G \quad$ gas constant

$h_{\mathrm{e}} \quad$ equivalent interfacial heat transfer coefficient for the liquid-vapor interface at the point of interest on the drop surface

Received on 12 August 1999 / Published online: 29 November 2001

C. Yamali $(\square)$

Department of Mechanical Engineering

Middle East Technical University

İnönü Bulvari, Ankara, 06531, Turkey

H. Merte Jr

Department of Mechanical Engineering and Applied Mechanics The University of Michigan

Ann Arbor, Michigan 48109, USA $h_{f g}$ $k$ $K_{1}$ $K_{2}$ $K_{\text {vol }}$ $n$ $n_{\text {nuc }}$ $N$ $N_{\text {co }}$ $N_{\mathrm{dc}}$ $p$ $p$
$P_{\mathrm{v}}^{*}$ 9 $q_{\mathrm{av}}^{\prime \prime}$ $q_{\mathrm{co}}^{\prime \prime}$

$r^{*}$

$r_{\mathrm{co}}$

$r_{\mathrm{co}}^{*}$

$r_{\mathrm{cr}}$

$r_{\mathrm{cr}}^{*}$

$r_{\text {dep }}$

$r_{\max }$

$R$

$t$

$T_{\mathrm{i}} \quad$ liquid-vapor interface temperature

$T_{s} \quad$ condenser surface temperature

$T_{\text {sat }}$ vapor saturation temperature

$T_{\mathrm{v}} \quad$ vapor temperature

$V_{\text {drop }}$ volume of a droplet

$y$

latent heat of vaporization drop thermal conductivity variable defined by Eq. (4) Eq. $(5)$ exponent used in Eq. (1) number of nucleation sites per unit area number of droplets tion range temperature total heat transfer total heat transfer rate through the droplet the droplet condensation heat flux without sweeping local heat flux on the condenser surface drop base radius nondimensional drop base radius start coalescing $\left(=r_{\mathrm{co}} / r_{\max }\right)$

base radius of a drop of critical size base radius of a departing droplet maximum drop size in the distribution point of interest fraction of number of droplets in coalescence range time period between two successive sweeping saturation pressure corresponding to vapor average heat flux over the condenser surface contribution of the coalescing droplets to the dropwise condensation heat flux without sweeping mean heat flux over the solid surface contact area of dropwise condensation heat flux without sweeping mean radius at which droplets start coalescing nondimensional base radius of a drop of critical size the radius of curvature of the drop interface at the

Greek symbols

$\gamma \quad$ condensation coefficient

$v \quad$ velocity of falling droplets

$\theta \quad$ contact angle

$\rho_{1} \quad$ liquid density 
$\rho_{\mathrm{v}} \quad$ vapor density

$\sigma \quad$ surface tension

$\tau_{\mathrm{dc}} \quad$ time period elapsed for the droplet to grow from critical size to $r_{\text {co }}$.

$\tau_{\mathrm{co}} \quad$ time period elapsed for $r_{\max }$ to grow from $r_{\mathrm{co}}$ to $r_{\mathrm{dep}}$

$\tau \quad$ total growth period

\section{1}

\section{Introduction}

Dropwise condensation, which was studied methodically first by Schmidt et al. [1], gives a condensation heat transfer coefficient an order of magnitude larger than can be obtained by filmwise condensation. Nevertheless, a successful application of dropwise condensation in heat exchanging equipment has not yet become a reality because no satisfactory means of sustaining dropwise condensation over a long period of time could be found. Elimination of the difficulties encountered in promoting dropwise condensation on a condenser surface requires a clear understanding of the mechanism of the dropwise condensation, which requires additional experimental and theoretical work.

Examination under a microscope of the surface on which dropwise condensation is taking place showed that droplets are not uniform in size, but vary over an extremely wide range. Considering these large variations in drop sizes, Lefevre and Rose [2] devised a new approach in predicting the dropwise condensation heat transfer. The theory presented in that work examines the heat transfer through a single drop and then calculates the average heat transfer rate by integrating over the assumed dropsize distribution:

$f_{\text {co }}\left(\frac{r}{r_{\max }}\right)=1-\left(\frac{r}{r_{\max }}\right)^{n}$

Similar approaches are made by other investigators to predict the heat transfer rates in dropwise condensation $[3,4]$.

Sweeping by departing drops was considered to have a significant effect on the heat transfer rate, even in the earliest works on dropwise condensation. Fatica and Katz [5] state that departing droplets originate in a region confined to within $1 / 4$ inch to $1 / 2$ inch from the top of the condensing surface. Therefore, as one moves downward on a condenser surface, the surface will be swept faster because of the increasing number of departing droplets. In the work of Fatica and Katz [5], it was speculated that the dropwise condensation heat transfer coefficient would be low in a region close to the top of the condenser surface, then will increase further down as a result of faster sweeping, then level off, and finally, as one proceeds sufficiently far down the surface, the surface will be flooded by falling droplets, and the heat transfer coefficient would decrease. This argument is supported by the work of Shea and Krase [6], who showed that the local heat transfer coefficient decreases beyond 4 inches from the top of a vertical cooling surface.

Intuition and the experimental results mentioned above show that the sweeping process is quite important in modeling and formulating dropwise condensation, and due care should be taken to isolate it from the other events taking place.

\section{2}

\section{Analysis}

Micro-cine studies of McCormick and Westwater [7], Peterson and Westwater [8] and the experimental studies of Umur and Griffith [9] and Ivanovskii et al. [10] show that the droplets form by nucleation process at specific locations called nucleation sites. Droplets so formed at these nucleation sites, which are usually pits and surface scratches, grow by direct condensation of vapor on them. These droplets fixed to the nucleation sites are called primary droplets, and since the critical size of these nucleating droplets are much smaller than the average distance between nucleation sites, they are far apart from one another. As the droplets grow they begin to coalesce, and their physical location is no longer limited to specific nucleation sites on the surface. The droplets move about as a result of coalescences, and can be located at virtually any point on the solid surface, occupying as much surface area as may be permitted by the capillary attraction forces between the droplets. Once the coalescences between droplets begins they grow both by direct condensation on the liquid/vapor interface and by random coalescences. These random coalescences, at some location on the surface, produces a droplet corresponding to the departure size, which then starts to slide down and sweeps the droplets on its path. On the clean path swept by the departing droplet, new droplets nucleate and begin to grow, again first by direct condensation and then by direct condensation and coalescences, until the dropsize distribution in the swept region becomes identical with the unswept region. The region is swept by another departing droplet and the cycle continues repetitively.

The analytical model to be presented below is based on the description of the events above, and especially emphasizes the following points:

1. In dropwise condensation the majority of the heat transfer in a drop takes place within a narrow region close to the perimeter of the drop, due to the very small thermal resistance existing here [11].

2. The majority of the heat transfer in dropwise condensation takes place through droplets of very small sizes [12], because of their small thermal resistances and large numbers.

\section{1}

\section{Conduction through droplets}

To calculate the heat transfer in dropwise condensation, it is essential to formulate the heat transfer through individual droplets. Krischer and Grigull [13], taking advantage of the relatively large heat transfer at the edge of the droplet, obtained a simple relation for the drop heat flux. On the other hand, Umur and Griffith [9] solved the conduction equation for a hemispherical droplet and obtained an exact solution for the drop heat flux. Both of the solutions mentioned above assumed a uniform interfacial heat transfer coefficient over the liquid vapor interface, and a uniform base temperature is taken. The assumption 
of a uniform interfacial heat transfer coefficient results in significant errors in the heat flux calculations, especially at drop sizes close to the critical size where the majority of the heat transfer takes place. Yamali and Merte [14] approximated the heat flow lines near the edge of the droplet by arcs of circles, and taking an interfacial heat transfer coefficient that varies with interface temperature and a constant base temperature, obtained the following relation for the drop heat flux:

$$
\begin{aligned}
& q_{\mathrm{d}}^{\prime \prime}(r)=\frac{q_{\mathrm{d}}(r)}{\pi r^{2}}=\frac{2 K_{1} B}{r^{2} T_{\mathrm{sat}}}\left(r \Delta T_{t}-K_{2} \sin \theta\right) \\
& x\left[-\cos \theta+\frac{r+B \cos \theta}{r} \ln \frac{r+B}{B}\right]
\end{aligned}
$$

where the interfacial heat transfer coefficient is expressed by

$h_{\mathrm{e}}=\frac{K_{1}}{T_{\mathrm{i}}}\left(1-\frac{K_{2}}{R \Delta T_{t}}\right)$

and

$K_{1}=\left(\frac{2 \gamma}{2-\gamma}\right)\left(\frac{1}{2 \pi}\right)^{1 / 2} \frac{h_{f g}^{2} P_{\mathrm{v}}^{*}}{G^{3 / 2} T_{\mathrm{sat}}^{3 / 2}}$

$K_{2}=\frac{2 T_{\mathrm{sat}} \sigma}{h_{f g} \rho_{\ell}}$

$\Delta T_{t}=T_{\mathrm{sat}}-T_{\mathrm{s}}$

$B=\frac{k T_{\text {sat }}}{\theta K_{1}}$

Knowing that Eq. (2) gives a better approximation to the drop heat flux, especially at small drop sizes, it will be used to calculate the dropwise condensation heat flux in this work.

\section{2}

\section{Heat flux in dropwise condensation excluding the effect of sweeping}

As one drop sweeps a surface it leaves a dry path behind. New droplets then nucleate on this path and grow randomly, first by direct condensation and at a later stage by coalescences as well. Since in principle the departing droplets may be removed from the surface randomly, the drop size distributions in the swept paths will be at different stages of development (i.e. the average drop size or the maximum drop size in a path may differ from one path to another). Whatever the development stage is, the drop size distribution in a swept path can be expressed by a unique relation in which the drop sizes are nondimensionalized by a characteristic drop size (e.g. maximum drop size). The form of this relation (Eq. (1)) is determined by close packing of circles on a surface, and whatever the characteristic drop size in the distribution is, it should always have the same form and be independent of other factors such as heat flux, temperature, pressure etc. In the analytical models devised thus far $[2,12]$, an average drop size distribution curve that averages the size distribution in various paths was employed. In a typical dropwise condensation taking place on a vertical surface it would seem difficult to define a unique function called the "average drop size distribution curve", due to the fact that it should be a strong function of heat flux. An increase in the heat flux increases the number of departing droplets, and the faster sweeping will increase the percentage of smaller size droplets present. If the analysis is based on the drop size distribution in the individual swept tracks this difficulty will be eliminated. Such an approach will be followed here; i.e. the first heat flux in a particular swept area will be calculated as a function of the maximum drop size in the distribution. Then, an average heat flux will be found for the entire surface by considering the rate of sweeping at every point on the surface.

The drop size distribution on a single swept area will be called the "uniform dropsize distribution". The drop size distribution on an entire condensing surface with significant swept areas at different stages of development will be called the "average dropsize distribution". The uniform dropsize distribution embodies two dropsize ranges: (a) the range between the critical drop size and the size corresponding to the order of the distance between nucleation sites, where the drops grow by direct condensation only, defined as primary drops, and (b) the range between this latter size and the maximum droplet size, where the drops grow by the combination of coalescence and direct condensation.

\section{3}

\section{Heat flux in the direct condensation range}

The continuous coalescences taking place among droplets of sizes sufficient for coalescences to take place, and the resulting motion associated with these coalescences, provide cleared surfaces on which fresh nucleation takes place, and new droplets form continuously on these available sites, randomly. Therefore, one can find drops of all sizes within the range corresponding to the direct condensation region, described as (a) in 2.2 above. From logical intuition one can say that the number of droplets that one can find in a given size range is proportional to the amount of time that the drop spends in that size range. Consequently, the dropsize distribution for the direct condensation range depends on the growth rate and the total time required for a drop to grow from the critical size up to the size on the order of the distance between the nucleation sites, i.e. its growth period.

\subsection{1 \\ Growth period}

The drop growth rate can be found from the equality of the rate of increase of the mass of the droplet and rate of condensation on its surface:

$$
\frac{\mathrm{d}}{\mathrm{d} t}\left(\rho_{\ell} V_{\mathrm{drop}}(r)\right)=\frac{\pi r^{2}}{h_{f g}} q_{\mathrm{d}}^{\prime \prime}(r)
$$

A geometrical expression is available that gives the drop volume as a function of the drop radius $r$ and the contact angle $\theta$, as:

$$
V_{\text {drop }}(r)=K_{\mathrm{vol}} r^{3}
$$

where

$$
K_{\mathrm{vol}}=\frac{\pi}{3 \sin ^{3} \theta}\left[2(1-\cos \theta)-\sin ^{2} \theta \cos \theta\right]
$$


Substituting Eq. (9) into Eq. (8) and taking the derivative:

$3 \rho_{\ell} K_{\mathrm{vol}} \frac{\mathrm{d} r}{\mathrm{~d} t}=\frac{\pi}{h_{f g}} q_{\mathrm{d}}^{\prime \prime}(r)$

Rearranging and integrating Eq. (11) from the critical size to the drop coalescence size, the time period for the growth of an individual droplet in the direct condensation range can be found as:

$\tau_{\mathrm{dc}}=\frac{3 \rho_{\ell} K_{\mathrm{vol}} h_{f g} r_{\max }}{\pi} \int_{r_{\mathrm{cr}}^{*}}^{r_{\mathrm{co}}^{*}} \frac{\mathrm{d} r^{*}}{q_{\mathrm{d}}^{\prime \prime *}\left(r^{*}\right)}$

where $r^{*}$ is the nondimensional drop base radius $\left(=r / r_{\max }\right), r_{\max }$ the maximum drop base radius in the distribution and $q_{\mathrm{d}}^{\prime \prime *}\left(r^{*}\right) \equiv q_{\mathrm{d}}^{\prime \prime}(r)$

$r_{\mathrm{cr}}^{*} \equiv \frac{r_{\mathrm{cr}}}{r_{\max }}$

$r_{\text {co }}$ is the mean radius at which droplets start coalescing, $r_{\mathrm{co}}^{*}$ the nondimensional average half distance between nucleation sites $\left(=r_{\mathrm{co}} / r_{\max }\right)$. It is also the size of the droplet at which coalescences begin.

$r_{\mathrm{cr}}=R^{*} \sin \theta$

where $R^{*}$ is given by

$R^{*}=\frac{2 T_{\text {sat }} \sigma}{h_{f g} \rho_{\ell} \Delta T_{t}}$

Equation (14) results from the relation between $R$ and $r$ as shown in Fig. 1.
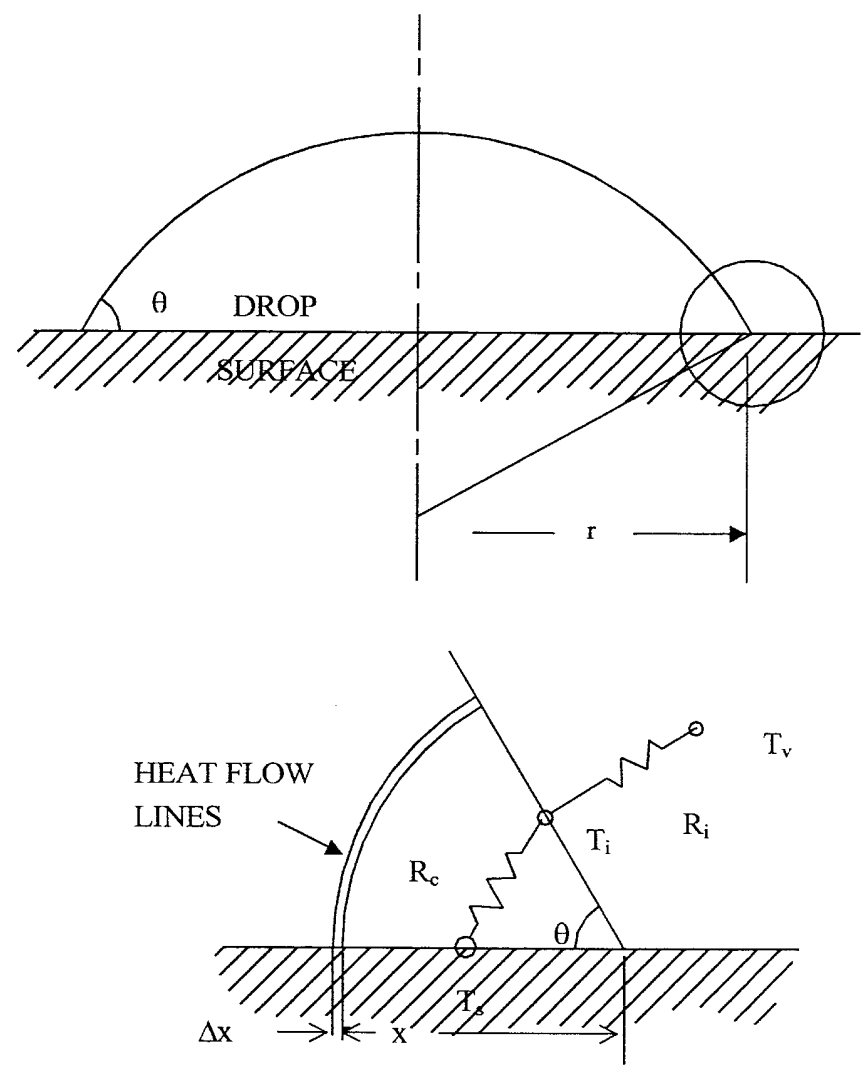

Fig. 1. Model for heat conduction through a droplet

\subsection{2}

\section{Drop size distribution}

Since the fraction of drops in a certain size range is proportional to the fractional time spent in that size range

$\frac{N\left(\Delta r_{1}\right)}{N_{\text {total }}}=\frac{\Delta t_{1}}{\tau_{\mathrm{dc}}}$

where $N\left(\Delta r_{1}\right)$ is the number of droplets in size range $\Delta r_{1}$, $N_{\text {total }}$ the total number of droplets in the direct condensation range, $\Delta t_{1}$ the time period elapsed during the growth of the droplet in size range $\Delta r_{1}$ and $\Delta t_{2}$ the time period elapsed during the growth of the droplet in size range $\Delta r_{2}$.

From the Eqs. (11), (12) and (16), the fraction of the droplets in the size range $r$ to $r+\mathrm{d} r$ can be obtained as

$\frac{N\left(d_{r}\right)}{N_{\text {total }}}=\frac{\mathrm{d} r^{*}}{q_{\mathrm{d}}^{\prime \prime *}\left(r^{*}\right) \int_{r_{\mathrm{cr}}^{*}}^{r_{\mathrm{co}}^{*}} \frac{\mathrm{d} r^{*}}{q_{\mathrm{d}}^{\prime \prime}\left(r^{*}\right)}}$

In order to find the total heat flux (based on the unit condenser area) in the direct condensation range the number of droplets per unit condenser surface area must be known. This can be expressed in terms of the fraction of droplets in size range $r$ to $r+\mathrm{d} r$ as

$$
\begin{aligned}
& {\left[\begin{array}{l}
\text { Fractional area occupied by primary } \\
\text { droplets in size range } r \text { to } r+\mathrm{d} r
\end{array}\right]} \\
& =\left[\begin{array}{l}
\text { Fraction of droplets } \\
\text { in size range } r \text { to } r+\mathrm{d} r
\end{array}\right] \\
& \times\left[\begin{array}{l}
\text { Number of nucleation } \\
\text { sites per unit area }
\end{array}\right] \\
& \times\left[\begin{array}{l}
\text { Base area } \\
\text { per drop }
\end{array}\right] \times\left[\begin{array}{l}
\text { Fractional area not occupied } \\
\text { by coalescing droplets }
\end{array}\right]
\end{aligned}
$$

The number of nucleation sites per unit area can be estimated by assuming a square matrix of nucleation sites, the distance between nucleation sites being $d_{\text {ns }}$. Number of nucleation sites per unit area then becomes

$n_{\mathrm{nuc}}=\frac{1}{d_{\mathrm{ns}}^{2}}=\frac{1}{4 r_{\mathrm{co}}^{2}}$

In reality, of course, the nucleation sites are randomly distributed and do not form a square matrix. This fact does not change the form of the Eq. (18) except the constant 4 may perhaps be slightly different.

The fractional area of the total condensing surface available for the primary or nucleating droplets is a fixed fraction of the area remaining outside the circular contact area with the condensing surface, of the coalescencing droplets of various sizes. During the course of the dropwise condensation cycle, the available area in any particular location might change materially as the droplets move, but its proportion should remain fixed and is given by the expression for the drop size distribution in the coalescence region (Eq. (1)) as $r_{\mathrm{co}}^{* n}$. The fractional area occupied by the primary droplets in size range $r$ to $r+\mathrm{d} r$ then becomes: 


$$
\begin{aligned}
\mathrm{d} f_{\mathrm{dc}} & =\frac{\mathrm{d} t}{\tau_{\mathrm{dc}}} \cdot n_{\mathrm{nuc}} \cdot \pi r^{2} \cdot r_{\mathrm{co}}^{* n} \\
& =\frac{r_{\mathrm{co}}^{* n}}{q_{\mathrm{d}}^{\prime \prime *}\left(r^{*}\right) \int_{r_{\mathrm{cr}}^{\mathrm{co}}}^{r_{\mathrm{c}}^{*}} \frac{\mathrm{d} r^{*}}{q_{\mathrm{d}}^{\prime \prime *}}\left(r^{*}\right)} \frac{\pi r^{2}}{4 r_{\mathrm{co}}^{2}} \mathrm{~d} r^{*}
\end{aligned}
$$

Finally, the drop size distribution function for the direct condensation region, defined as the number of droplets in size range $\mathrm{d} r^{*}$ (or $\mathrm{d} r$ ) per unit condenser area $\left(A_{\mathrm{c}}\right)$ becomes:

$\frac{\mathrm{d} N_{\mathrm{dc}}}{A_{\mathrm{c}}}=\frac{\mathrm{d} f_{\mathrm{dc}}}{\pi r^{2}}$

Substituting Eq. (19) into Eq. (20) and rearranging, the number of droplets in size range $\mathrm{d} r^{*}$ per unit nondimensional condenser area $\left(A_{c} / r_{\max }^{2}\right)$ is expressed as:

$$
\frac{\mathrm{d} N_{\mathrm{dc}}}{A_{\mathrm{c}} / r_{\max }^{2}}=\frac{r_{\mathrm{co}}^{* n-2} \mathrm{~d} r^{*}}{4 q_{\mathrm{d}}^{\prime \prime *}\left(r^{*}\right) \int_{r_{\mathrm{cr}}^{*}}^{r_{\mathrm{c}}^{*}} \frac{\mathrm{d} r^{*}}{q_{\mathrm{d}}^{\prime \prime *}\left(r^{*}\right)}}
$$

\subsection{3}

\section{Net heat flux}

The heat flux in the direct condensation region can be found by integrating the heat flux through individual droplets over the entire drop population of the direct condensation region:

$q_{\mathrm{dc}}^{\prime \prime}=\int_{r_{\mathrm{cr}}^{*}}^{r_{\mathrm{cc}}^{*}} q_{\mathrm{d}}^{\prime \prime *}\left(r^{*}\right) \mathrm{d} f_{\mathrm{dc}}$

Substituting $\mathrm{d} f_{\mathrm{dc}}$ from Eq. (19) into Eq. (22) and integrating:

$q_{\mathrm{dc}}^{\prime \prime}=\frac{\pi}{12} r_{\mathrm{co}}^{* n-2} \frac{r_{\mathrm{co}}^{* 3}-r_{\mathrm{cr}}^{* 3}}{\int_{r_{\mathrm{cr}}^{*}}^{r_{\mathrm{co}}^{*}} \frac{\mathrm{d} r^{*}}{q_{\mathrm{d}}^{* \prime}\left(r^{*}\right)}}$

\section{4}

\section{Heat flux in the coalescence range}

The dropsize distribution of coalescing droplets can be expressed by a function of the form given by Eq. (1), and conforms well to experimental measurements for values of $n$ close to $1 / 3$ [15]. $f_{\text {co }}$ in Eq. (1) is the fraction of area occupied by droplets in the size range from a radius $r$ to the maximum radius $r_{\max }$ in the distribution. Variations in the experimental values of $n$ given in the literature are speculated to be due to the sweeping of the surface by falling droplets. The magnitude of the sweeping effect varies with the heat flux, and this is reflected in variations of the dropsize distribution.

The size distribution given by Eq. (1) applies for coalescing droplets only because measurements have been made on these types of droplets. Droplets growing by direct condensation are too small to be counted by the naked eye or even under a microscope. Consequently, Eq. (1) applies for droplets from the maximum size (departure size) down to the radius order of magnitude of half the distance between nucleation sites.

The fraction of area occupied by droplets in the coalescence region, within the dimensionless differential size range $\mathrm{d} r^{*}$ becomes, from Eq. (1): $\mathrm{d} f_{\mathrm{co}}=n r^{* n-1} \mathrm{~d} r^{*}$

The contribution to the total heat flux due to those drops in the coalescence region then can be found by integrating the heat conduction through the individual droplets over the entire population of coalescing droplets:

$q_{\mathrm{co}}^{\prime \prime}=\int_{r_{\mathrm{co}}^{*}}^{1} q_{\mathrm{d}}^{\prime \prime *}\left(r^{*}\right) n r^{* n-1} \mathrm{~d} r^{*}$

The total heat flux for dropwise condensation excluding the sweeping effect (e.g. dropwise condensation on a facedown surface) is the sum of the sequential contributions due to the coalescing drops (Eq. (25)) and the drops growing by direct condensation (Eq. (23)):

$q_{T}^{\prime \prime}=q_{\mathrm{dc}}^{\prime \prime}+q_{\mathrm{co}}^{\prime \prime}$

\section{5 \\ Heat flux in dropwise condensation including the effect of sweeping}

Equation (26) provides the heat flux for dropwise condensation as a function of the maximum drop size in the distribution, but excludes the effect of sweeping of the surface by the departing droplets. In the definition of Eq. (26) it was assumed that droplets reaching the departure size were removed without having any direct influence on neighboring areas. An example for this situation might be dropwise condensation taking place on a horizontal surface facing downward. The heat flux on a swept track following a falling droplet can also be calculated by Eq. (26) except that, unlike the case of the downward facing surface, the maximum drop radius in the swept area is smaller than the departure size, and is now a function of time. The heat flux therefore changes in decreasing manner as time passes. Dropwise condensation on a vertical surface is nothing more than various swept regions with varying maximum (or average) drop radii. With such a surface, one now can calculate the heat flux for the entire surface, using Eq. (26) and a computed maximum drop size in each swept area.

The sweeping of the surface is affected by the height of the condenser surface; no sweeping takes place at the top of the condenser surface, while the sweeping rate can be expected to increase at the lower parts of the surface, since more and more departing droplets join the falling droplets. Therefore, the frequency of sweeping at any height can be calculated by summing the number of departing droplets generated at any point on the surface over the height of the condenser surface to that point.

Here, a new parameter $f_{\mathrm{d}}(y)$ will be introduced, which can be described as the number of drops attaining the departure size, per unit time, per unit area, at a height $y$. If a differential horizontal strip on the condenser surface is taken, (Fig. 2), one will see the formation and departure of departing droplets in a certain time interval that occupies a certain portion of the differential strip. Since the diameter of the departing droplets is larger than the differential strip, only a portion of the departing droplets are within the differential strip, and a problem exists as to how the number of drops within such a strip can be counted. This 


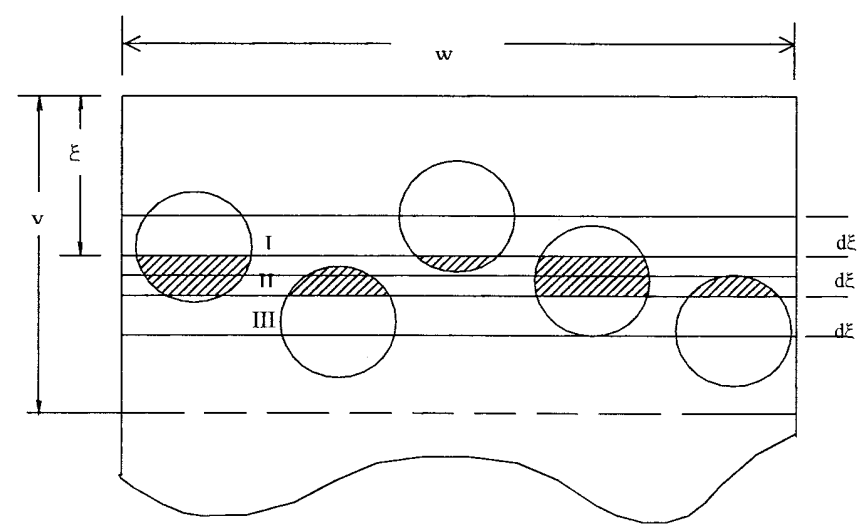

Fig. 2. Model for determining the local number of departing drops on a vertical surface

difficulty can be circumvented in one of two ways: (a) a departing droplet is assumed to belong to a differential strip if its center is located precisely in the particular strip just before it begins to fall, and the number of departing droplets that belong to this strip are the total number of drop centers within the strip, or (b) the average number of departing droplets within this strip is obtained by dividing the total area of the departing droplets remaining in the differential strip just before they begin to fall by the base area of a single departing droplet. $f_{\mathrm{d}}(y)$, then, becomes the number so obtained divided by the area of the differential strip and the time interval elapsed.

The total number of departing droplets that cross a horizontal line at an altitude of $y$ per unit time can be obtained by integrating the local number over the condenser area above this line, as:

$f_{\mathrm{s}}(y)=w \int_{0}^{y} f_{\mathrm{d}}(\xi) \mathrm{d} \xi$

where $f_{s}(y)$ is the number of departing drops crossing elevation $y$, per unit time, for a condenser surface of width $w$.

It is necessary to determine the frequency at which a point on the condenser surface is swept, since this frequency affects the rate of heat transfer at that point. The time interval between two successive sweepings at a particular point on the condenser surface will be defined as the sweeping period, and can be derived in terms of the other parameters by using the model illustrated in Fig. 3. The condenser surface is divided into vertical strips of width $D_{\text {dep }}$, which is taken as the departure diameter of a drop, such that the point of interest, " $A$ ", lies at the center of one of the strips. As shown, departing droplets above line $y$ are randomly distributed over the strip such that on the average each strip has the same number of departing droplets, because of the random nature of dropwise condensation. Here again, it is necessary to describe carefully a strip having a departing droplet since, as is shown in Fig. 3, departing droplets most likely will be only partly contained within the strip under consideration. As a convention, a drop will be assumed to belong to a strip if its center is in that strip. The rate at which the number of
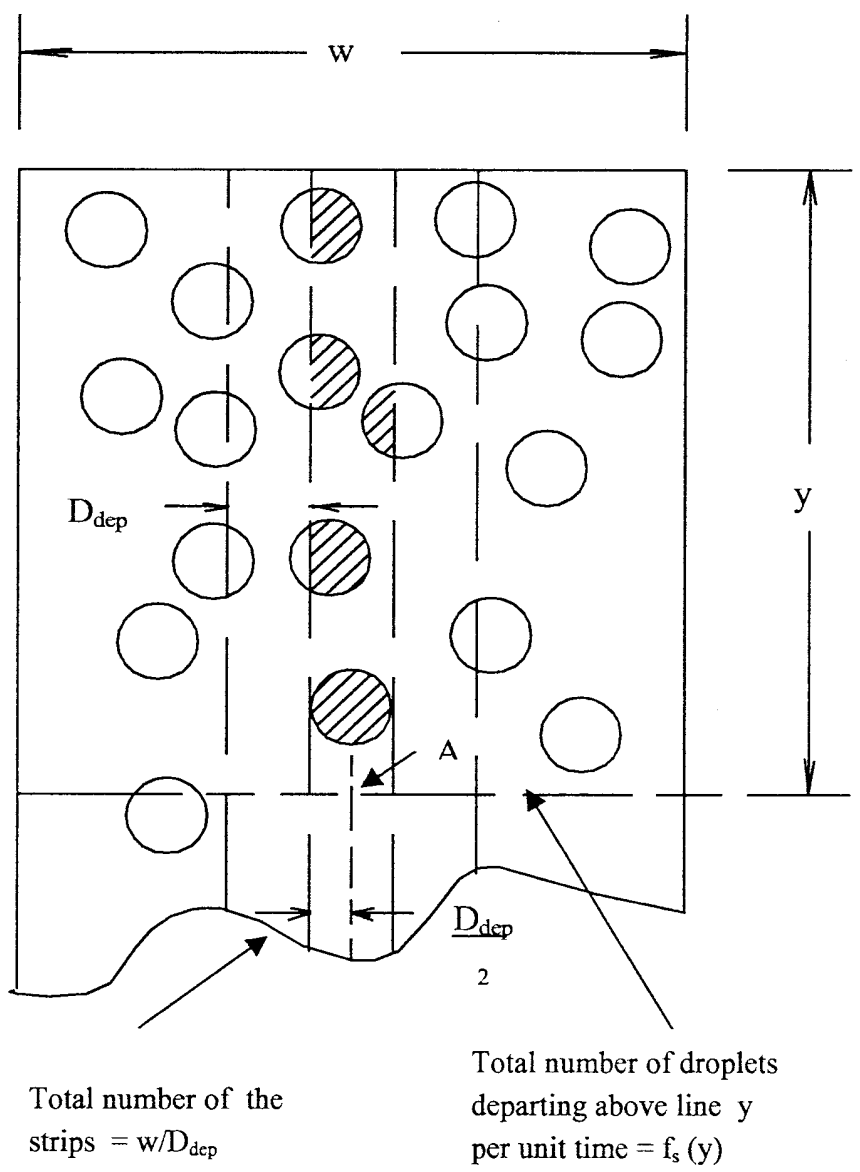

Fig. 3. Model for sweeping frequency

departing droplets is generated within a strip above line $y$ is then:

$N_{\text {dep }}(y)=\frac{f_{\mathrm{s}}(y)}{w / D_{\text {dep }}}=D_{\text {dep }} \int_{0}^{y} f_{\mathrm{d}}(\xi) \mathrm{d} \xi$

Since any droplet remaining in the strip above $y$ sweeps the middle point, the average sweeping period is given by:

$p(y)=\frac{1}{D_{\text {dep }} \int_{0}^{y} f_{\mathrm{d}}(\xi) \mathrm{d} \xi}$

In order to understand the events that take place between two successive sweepings at a particular point on the surface, various differential swept areas of height $\mathrm{d} \xi$ at a given elevation $y$ and at a given time $t$ following a sweep are placed side by side in Fig. 4 . The events between two successive sweepings are considered as made of three distinct phases.

In the first phase, which begins immediately after the sweeping $(t=0)$, the surface is completely bare (Fig. 4a). After a time interval drops nucleate and begin growing by direct condensation until the they reach size $r_{c o}$ in a time interval $t_{\mathrm{dc}}$ (Fig. $4 \mathrm{~b}$ ). In the second phase coalescences between adjacent drops then take place, and the drops continue to grow (Fig. 4c) until a departure size is reached (Fig. 4d). The time elapsed between the onset of coalescence and the next appearance of the first departing droplet will be called $t_{\mathrm{co}}$. Therefore, the time elapsed between the 


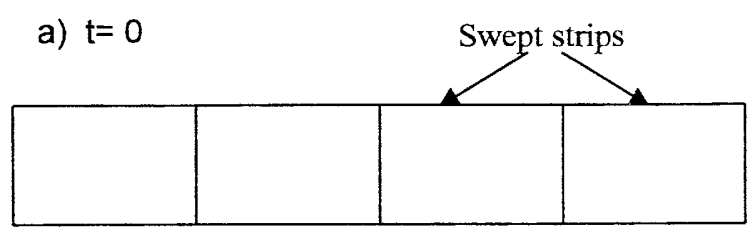

b) $0<t<\tau_{d c}$

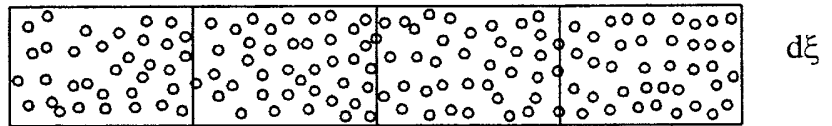

c) $\tau_{\mathrm{dc}}<\mathrm{t}<\tau$

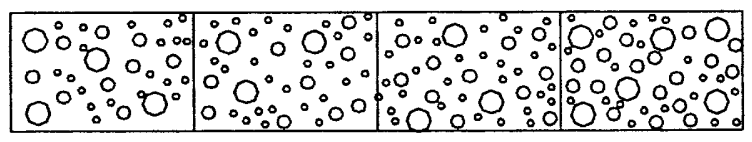

$\mathrm{d} \xi$
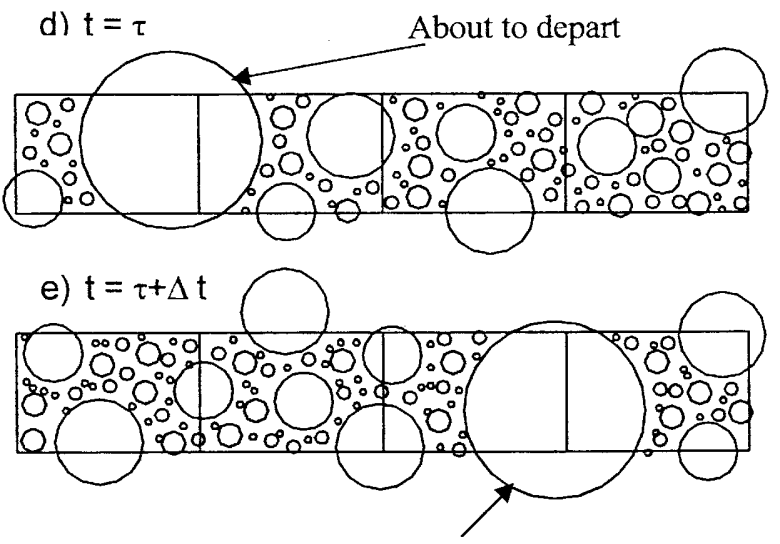

About to depart

Fig. 4. Typical behavior at a horizontal strip between successive sweepings

sweeping and the next appearance of a departing droplet will be defined as the total growth period, given as:

$\tau=\tau_{\mathrm{co}}+\tau_{\mathrm{dc}}$

In the third phase, the drop size distribution reaches a steady function and will not change any further as can be seen in Fig. (4d) and Fig. (4e). A drop has departed between Fig. (4d) and Fig. (4e), and new drops nucleate within the area cleared. At the same time other drops continue to grow by coalescences and direct condensation, and another drop at another location reaches the departure size. This description of the phenomena taking place between two successive sweepings demonstrates that a certain time interval $(\tau)$ is required for droplets in a swept area to attain the same size distribution as the droplets in the unswept areas. Only after this time interval can the swept region again participate in generating departing droplets.

If no sweeping were to take place, $f_{\mathrm{d}}(y)$ on a differential strip as in Fig. (4d) and (4e) would be given by:

$f_{\mathrm{d}}(y)=q_{T}^{\prime \prime}\left(r_{\mathrm{dep}}\right) / m_{\mathrm{d}} h_{f g}$

where $m_{\mathrm{d}}$ is the mass of a departing droplet. Because of the sweeping, however, no departure sized drops can be produced within the differential strip during the total growth period $t$. Only within the time interval $(p-t)$ can departure sized droplets be generated in a differential strip. Therefore, in order to obtain a time average of the function $f_{\mathrm{d}}(y)$ over a sweeping cycle, Eq. (31) must be multiplied by the factor $(p(y)-\tau) / p(y)$.

Because of their relatively large size, the sweeping droplets have a very large thermal resistance and, therefore, the condenser surface that remains under them as they sweep the surface can be considered as insulated. If the average number of falling droplets per unit area is given by $D_{\mathrm{d}}(y)$, then the fraction of the area not covered by these droplets becomes $\left(1-D_{\mathrm{d}}(y) D_{\mathrm{dep}}^{2} \pi / 4\right)$. The expression that gives $f_{\mathrm{d}}(y)$ (Eq. (31)) is then corrected for this blanketing effect and for its time average, giving:

$f_{\mathrm{d}}(y)=\frac{\left[1-D_{\mathrm{d}}(y) \frac{\pi}{4} D_{\mathrm{dep}}^{2}\right]\left(\frac{p(y)-\tau}{p(y)}\right) q_{T}^{\prime \prime}\left(r_{\mathrm{dep}}\right)}{m_{\mathrm{d}} h_{f g}}$

The number of falling droplets in motion per unit condenser surface area at height $y$ can be expressed as:

$D_{\mathrm{d}}(y)=\frac{f_{\mathrm{s}}(y)}{v w}$

where $v$ is the velocity of the falling droplets.

From the balance of the viscous and gravity forces acting on a falling droplet $v$ can be obtain as:

$v \propto \frac{D_{\mathrm{dep}}^{2} g \cdot \rho_{\ell}}{\mu}$

where $\mu$ is the condensate viscosity. [16]:

By dimensional analysis and also from the analysis in

$D_{\text {dep }} \propto \frac{1}{\sqrt{g}}$

Substituting Eq. (29) and (33) into Eq. (32), the time average sweeping frequency is obtained as an integral equation:

$$
\begin{aligned}
f_{\mathrm{d}}(y)= & \frac{q_{T}^{\prime \prime}\left(r_{\mathrm{dep}}\right)}{m_{\mathrm{d}} h_{f g}}\left[1-\frac{\pi D_{\mathrm{dep}}^{2}}{4 v} \int_{0}^{y} f_{\mathrm{d}}(\xi) \mathrm{d} \xi\right] \\
& \times\left[1-\tau D_{\mathrm{dep}} \int_{0}^{y} f_{\mathrm{d}}(\xi) \mathrm{d} \xi\right]
\end{aligned}
$$

The analysis above assumes that $v$ is uniform and that the initial acceleration is negligible. The effect of the increase in size of the width of the swept path as the drop is falling is considered to be insignificant because of the elongation of the drop as it falls down, and because the increase in the drop dimension is approximately proportional to the cube-root of the increase in drop volume.

In Eq. (36), $\tau$ is given by Eq. (30), and $t_{\mathrm{dc}}$ is given by Eq. (12). $t_{\mathrm{co}}$ is the time period required for the maximum drop size in the population to reach $r_{\text {dep }}$ from the instant that droplets being coalescing, and can be found from the instantaneous mass balance of the condensate forming on the swept region and from the growth rate of the entire drop population, over the period from the onset of 
coalescence up to the time where the maximum drop size attains the departure size:

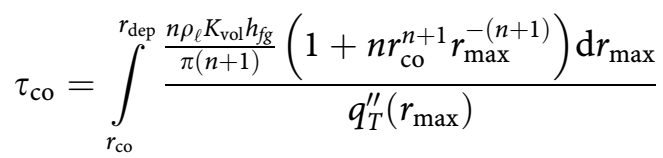

In the derivation of the Eq. (37), it is assumed that the mass of the primary droplets are negligible, for their size and the fraction of the condenser area occupied by them is negligibly small.

In the paragraphs surrounding Eq. (30), the events between successive sweepings are considered to consist of three distinct phases. Therefore, in calculating the total heat transfer rate on the surface, the heat flux in each phase should be calculated independently, followed by the computation of the time average over the entire sweeping period. Immediately following a sweeping action new drops nucleate and grow with no coalescences up to the size $r_{\mathrm{co}}$, within the time period $\tau_{\mathrm{dc}}$. Therefore, the total heat transfer to the condenser surface in the first phase can be calculated by multiplying the total mass of the droplets on the condenser surface, just before the coalescences begin, by the latent heat. Since the number of droplets just before the coalescences begin is $n_{\text {nuc }}$, from Eq. (18), the total heat transferred in phase I becomes:

$Q_{\mathrm{PI}}=h_{f g} m_{\mathrm{d}} n_{\mathrm{nuc}}=\frac{h_{f g} \rho_{\ell} K_{\mathrm{vol}} r_{\mathrm{co}}}{4}$

In the second phase, drops then begin to coalesce and grow until the size distribution is indistinguishable from the unswept area. During this second phase the maximum drop size and the average drop size increase. Consequently, the heat flux varies as time proceeds. The total heat transfer in the second phase can be obtained by integrating the heat flux given by Eq. (26) over the time interval $t_{\mathrm{co}}$ :

$Q_{\text {PII }}=\int_{\tau_{\mathrm{dc}}}^{\tau} q_{T}^{\prime \prime}\left(r_{\max }\right) \mathrm{d} t$

The total heat transfer in the third phase is the product of the length of the third phase and its heat flux. The heat flux is constant in this phase since the maximum drop size does not change.

The time averaged local heat flux can be found by summing the energy transfer to the condenser surface in the three phases, and then dividing the sum by the total period of elapsed time $(p)$. The value obtained by this operation should be multiplied by $\left[1-D_{\mathrm{d}}(y)(\pi / 4) D_{\mathrm{dep}}^{2}\right]$, the fraction of the condenser area not covered by falling droplets. This accounts for the fact that the falling droplets have relatively large thermal resistances, and therefore the area under them is insulated, for all practical purposes. The final expression for the time averaged local heat flux becomes:

$q_{\mathrm{loc}}^{\prime \prime}(y)=\frac{\left[1-D_{\mathrm{d}}(y) \frac{\pi}{4} D_{\mathrm{dep}}^{2}\right]}{p(y)}$ $x\left[\frac{h_{f g} \rho_{\ell} K_{\mathrm{vol}} r_{\mathrm{co}}}{4}+\int_{\tau_{\mathrm{dc}}}^{\tau} q_{T}^{\prime \prime}\left(r_{\mathrm{max}}\right) \mathrm{d} t+(p-\tau) q_{T}^{\prime \prime}\left(r_{\mathrm{dep}}\right)\right]$

It can be seen that $q_{\text {loc }}^{\prime \prime}$ is a function of the condenser height $y$. This is an expected result, since the condenser surface area is swept more frequently at the lower regions than at the upper regions. The average heat flux from the upper edge of the condenser surface to the height $y$ can be expressed as:

$q_{\mathrm{av}}^{\prime \prime}=\frac{1}{y} \int_{0}^{y} q_{\mathrm{loc}}^{\prime \prime}(\xi) \mathrm{d} \xi$

\section{Results}

The results of numerical calculations using the analytical model developed above are presented in Figs. 5 to 12, for the condensation of saturated steam at atmospheric pressure. It was necessary to determine one parameter empirically in order to proceed with the computations; the mean spacing between the nucleation sites $\left(2 r_{\mathrm{co}}\right)$, or the related nucleation site density. A single value was used in

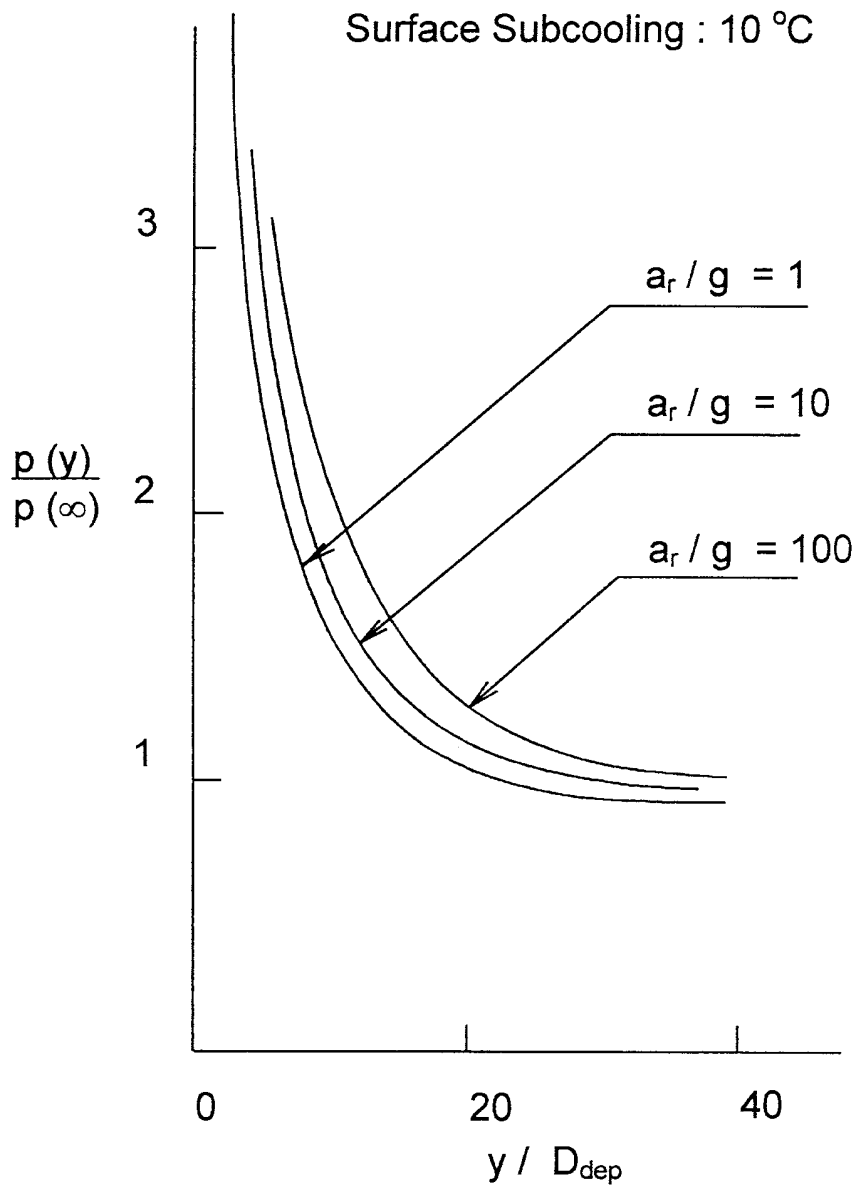

Fig. 5. Variation of nondimensional sweeping period with respect to the nondimensional surface height 


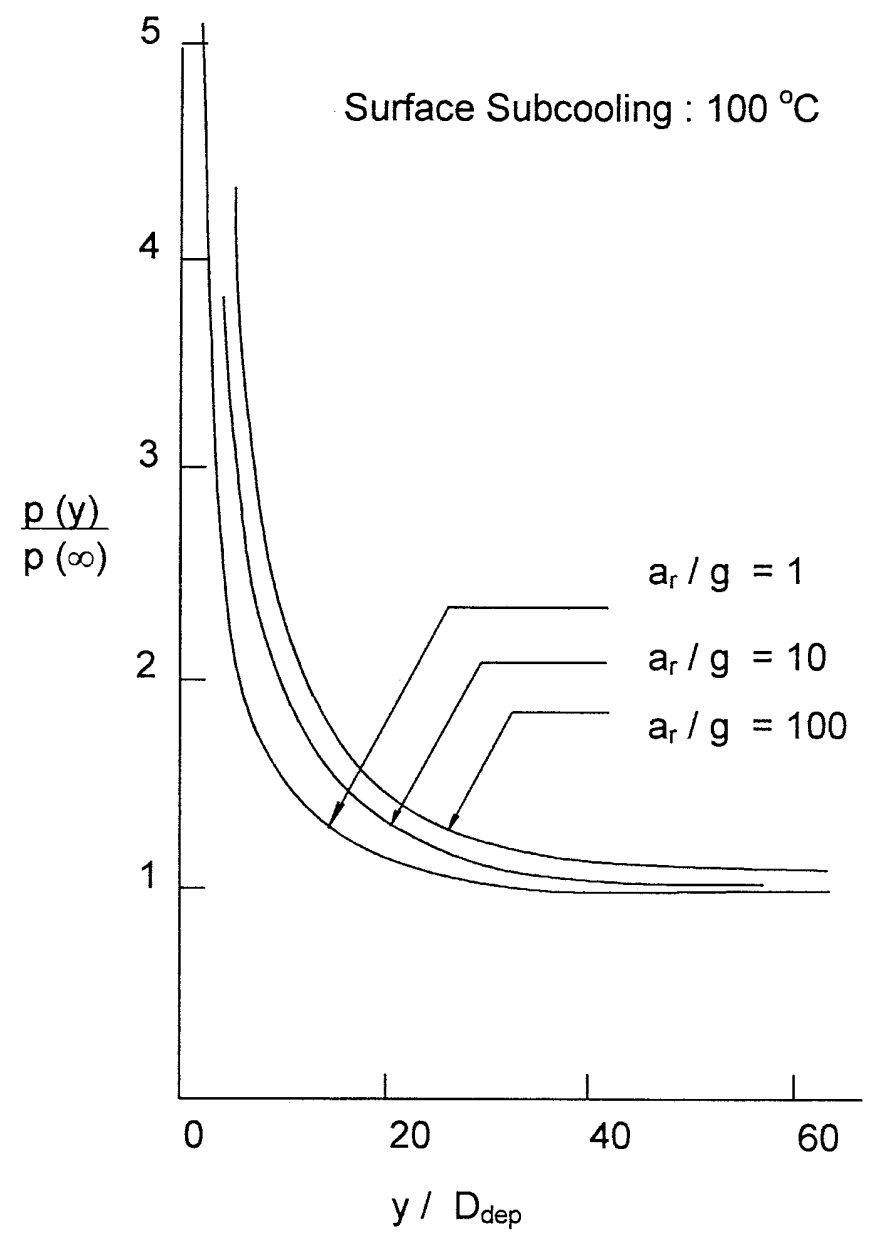

Fig. 6. Variation of nondimensional sweeping period with respect to the nondimensional surface height

all the computations performed here, $r_{\mathrm{co}}=2.6 \times 10^{-6} \mathrm{~m}$. This value of $r_{\mathrm{co}}$ is chosen to obtain the best fit with the experimental results presented in Ref. [17]. The contact angle is taken as constant at $65^{\circ}$. The condensation coefficient and the exponent $n$ in drop size distribution function for the coalescence region (in Eq. (1)) are taken as 1 and $1 / 3$, respectively. The velocity of the falling drops was taken as $0.6 \mathrm{~m} / \mathrm{s}$, obtained by measurement on vertical surfaces in earth gravity Ref. [18]. When computations are carried out for high gravity circumstances, this velocity would be expected to increase somewhat. However, the effect on the final results would be negligibly small, because of the relatively large velocity to begin with. The numerical calculations are carried out using an iteration process. A boundary value must be assumed for the local drop departure rate. The solution of the integral equation, Eq. (36), requires a boundary condition at $y=0$. This condition is obtained from the fact that no sweeping takes place at the upper edge of the vertical condensing surface $(y=0)$. The drop departure rate here is thus equal to the heat flux given by Eq. (26), divided by the latent heat and the mass of a single departing droplet. This value is also taken as the initial value for the local drop departure rate to be iterated in Eq. (36), since this is the largest value that the drop departure rate can attain, being the drop departure rate for an unswept area. The computation is carried

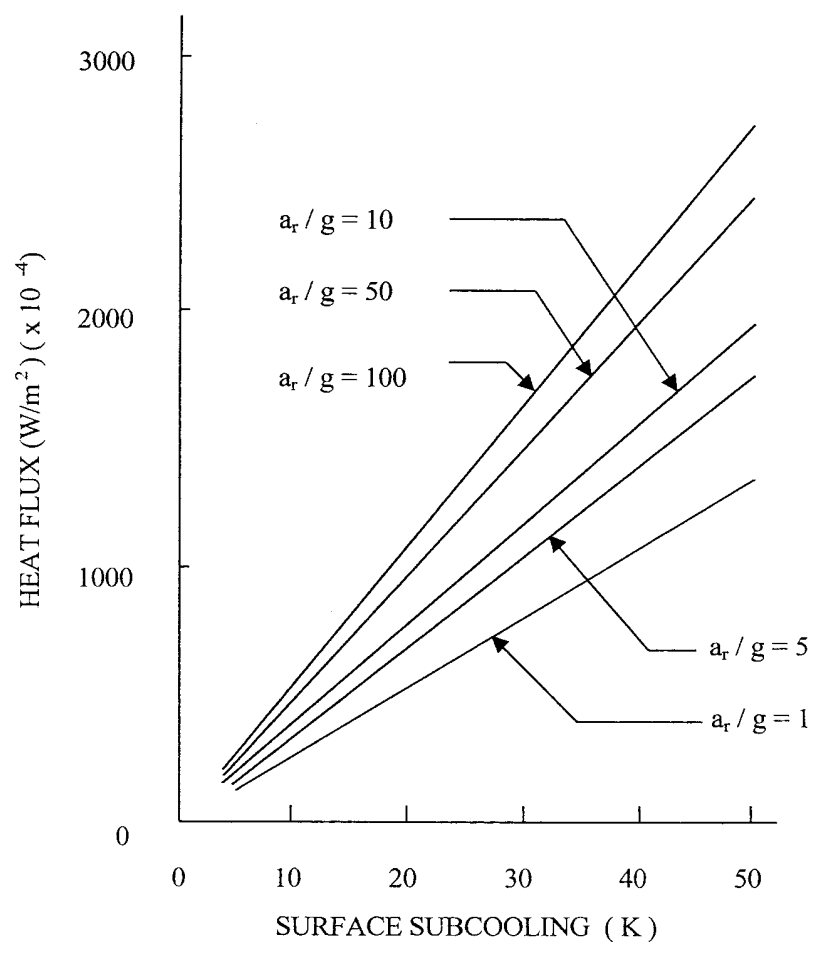

Fig. 7. Heat flux on an unswept area versus surface subcooling

out by substituting the assumed departure rate into Eq. (36) and iterating until the calculated values converge.

The variation of the sweeping period as a function of the distance from the upper edge for two different levels of surface subcooling $\left(10^{\circ} \mathrm{C}\right.$ and $\left.100{ }^{\circ} \mathrm{C}\right)$ and for departure sizes ranging from $a_{r} / g=1$ to $a_{r} / g=100$ are shown in Figs. 5 and 6 , respectively. In both figures, the distance from the upper edge and the sweeping period are nondimensionalized by the departure diameter $\left(D_{\text {dep }}\right)$ and the sweeping period at large distances from the upper edge, respectively, to provide a better comparison of the curves for various departure sizes. It can be seen that the sweeping period is very large at locations near the upper edge, and becomes smaller as $y / D_{\text {dep }}$ increases, and reaches an asymptotic value of 1 . The sweeping period is infinite at the upper edge, since no sweeping occurs at this location. The results show that the sweeping period is almost constant for large distances away from the upper edge. This is an expected result since the sweeping frequency increases as one goes down the surface, as more and more departing droplets join the falling droplets. However, this in turn decreases the number of droplets that reach the departure size. Figures 5 and 6 also show that for the values of $y / D_{\text {dep }}$ around 40 , the sweeping period reaches its asymptotic value for various departure sizes and surface subcoolings.

Comparison of Figs. 5 and 6 shows that although the departure size effects the sweeping period versus surface height behavior, this effect is not a significant one, especially at low surface subcooling levels. Therefore, it is possible to obtain a universal curve giving the nondimensional sweeping period in terms of the nondimensional surface height. 


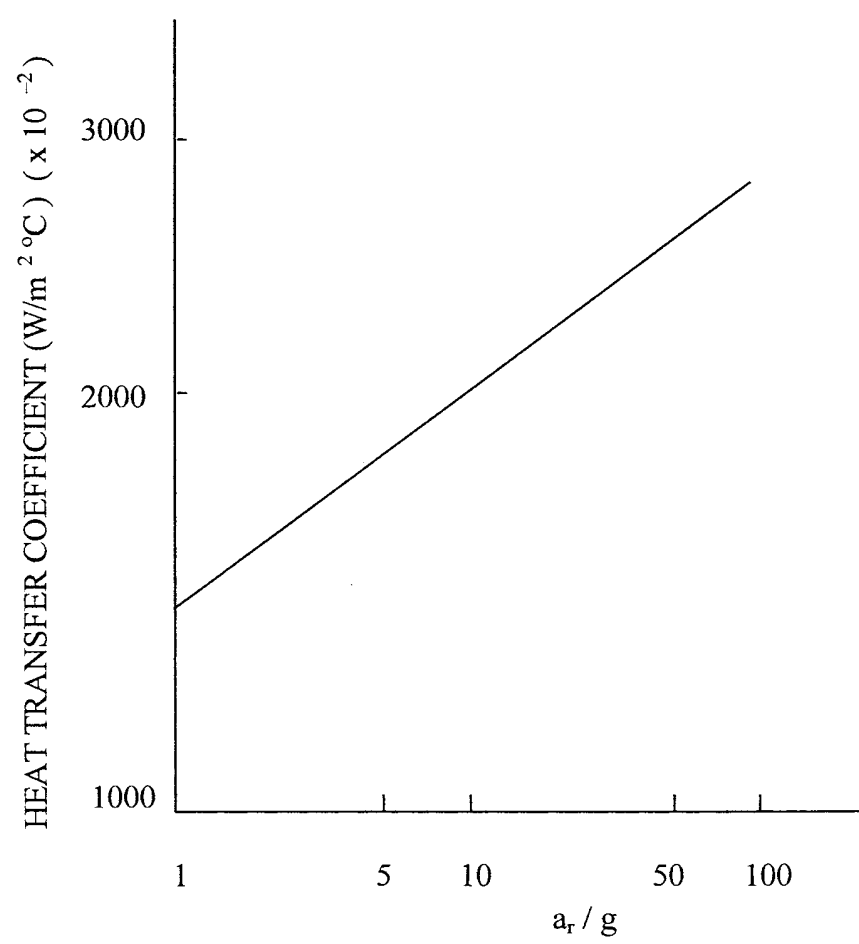

Fig. 8. Condensation heat transfer coefficient on an unswept area

The dropwise condensation heat flux for a non-swept surface (e.g. dropwise condensation on a horizontal facedown surface) is shown in Fig. 7 as a function of surface subcooling for various $a_{r} / g$ ratios. The nonswept surface heat flux is a linear function of the surface subcooling here, and the dropwise condensation heat transfer coefficient for such a surface is constant at the $a_{r} / g$ ratios shown.

Figure 8 presents the variation of the heat transfer coefficient on a nonswept condensing surface with respect to a $a_{r} / g$ ratio, using logarithmic axes, and shows that the variation is a straight line. This means that the heat transfer coefficient for dropwise condensation on a non-swept area can be expressed as:

$h=C\left(\frac{a_{r}}{g}\right)^{b}$

where $C$ and $b$ are constants and depend on the condensing fluid.

Figures 9 and 10 show the value of the local heat flux at $a_{r} / g=100$ and $a_{r} / g=1$, respectively, as a function of the distance from the upper edge of the vertical condensing surface for the various values of surface subcooling used. The distance from the upper edge and the local heat flux are nondimensionalized by the drop departure diameter and the unswept heat flux (heat flux at the upper edge), respectively, for a better comparison at various departure sizes and subcoolings. These show that the distance from the upper edge has a strong influence on the local heat flux. Whatever the values of the surface subcooling and $a_{r} / g$ ratio, the local heat flux converges to an asymptotic value at large distances from the upper edge. At the large body force in Fig. 9, corresponding to small departure sizes, an interesting behavior is observed. At large surface

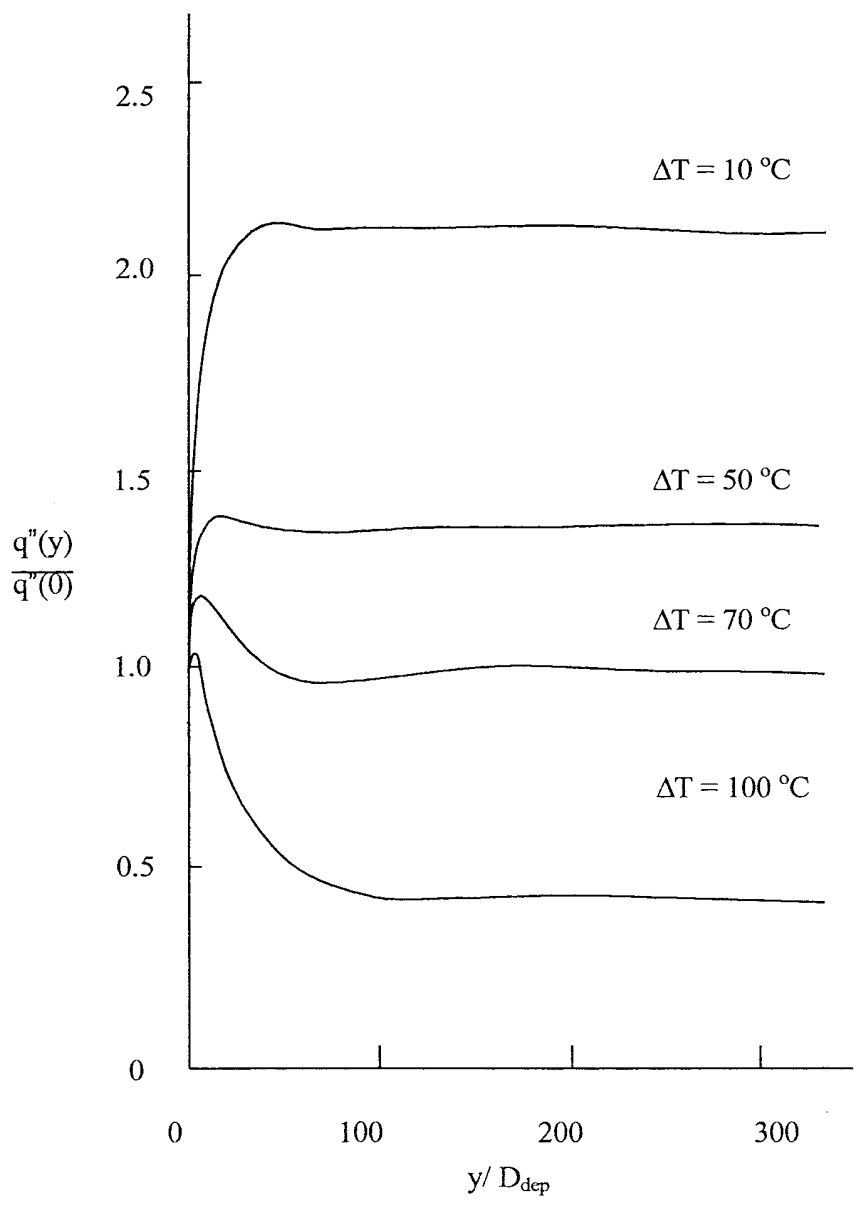

Fig. 9. Variation of nondimensional heat flux with respect to nondimensional height $\left(a_{r} / g=100\right)$

subcooling levels the local heat flux first increases and then decreases. The increase in the local heat flux near the upper edge of the vertical surface takes place as a result of faster sweeping of the surface. This increase in the sweeping frequency has an opposite effect on the local heat flux at large distances from the upper edge, since the surface becomes flooded by the large number of departing droplets there, insulating the surface and reducing the heat flux. At the low body force of $a_{r} / g=1$ in Fig. 10, corresponding to large departure sizes, the local heat flux increases monotonically with the distance from the upper edge. The frequency of sweeping increases with this distance, with the attendant increase in heat flux because of the smaller drop size, eventually reaching the asymtotic value as observed.

Figures 11 and 12 shows the variation of the mean heat flux on a vertical condensing surface of heights 0.01 and $0.1 \mathrm{~m}$, respectively, with respect to the surface subcooling at various $a_{r} / g$ ratios. It can be seen that the mean heat flux increases as surface subcooling increases, although the rate of increase becomes smaller at the larger surface subcoolings. The effect of the closely packed departing droplets associated with large surface subcoolings can be seen in Fig. 12 as a decrease in the mean heat flux at large body forces. Figure 12 clearly shows the existence of a peak heat flux for dropwise condensation, similar to the case of 


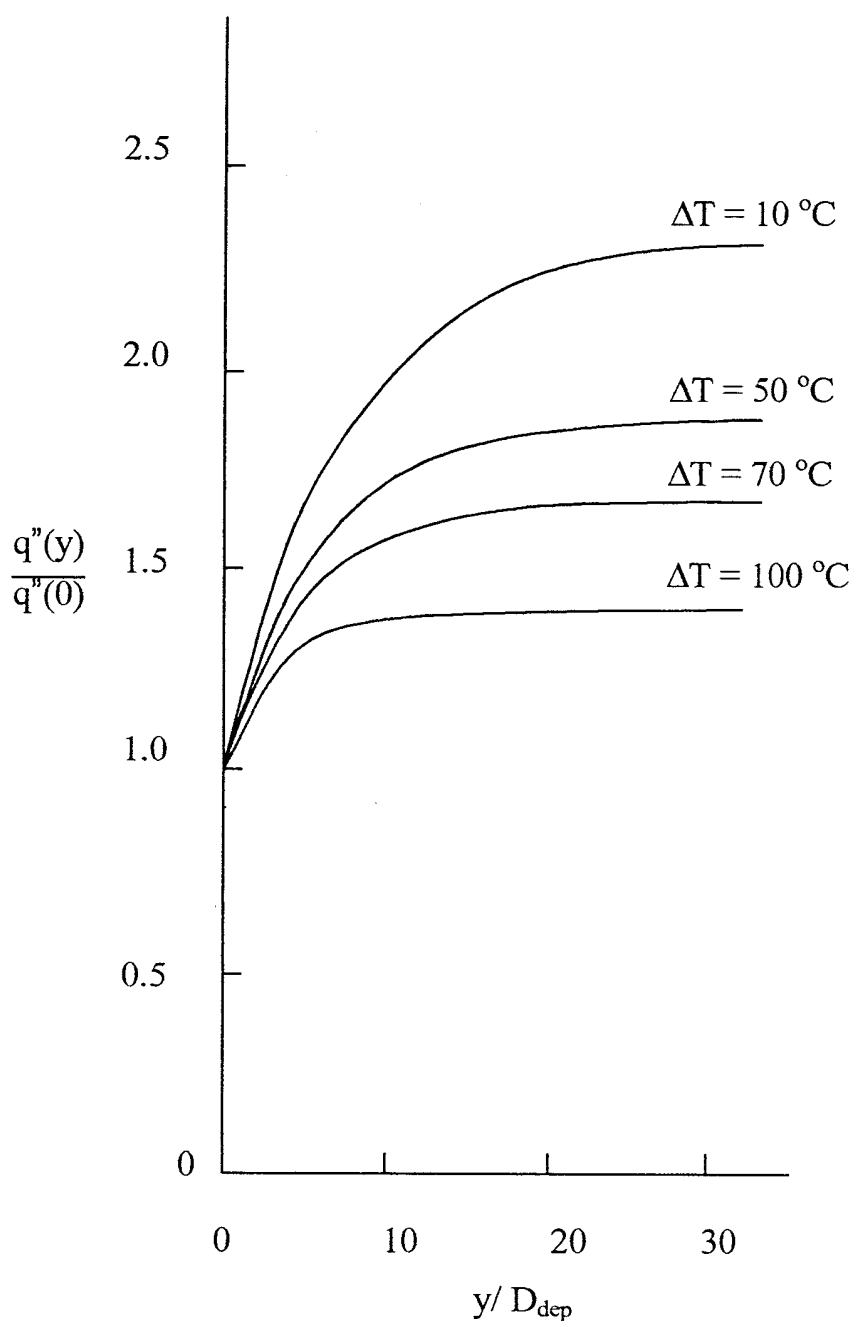

Fig. 10. Variation of nondimensional heat flux with respect to nondimensional height $\left(a_{r} / g=1\right)$

the critical heat flux in boiling heat transfer. Such a trend cannot be observed if the departure size is large and/or the plate height is small.

In Fig. 13, the predictions of the analytical model derived in this work are compared with the experimental measurements presented in Refs. 17 and 20. The condenser surface used in Ref. 17 is a circular horizontal surface, $1.9 \mathrm{~cm}$ in diameter, on which a centrifugal body force is acting parallel to the surface. Since the vertical mean heat flux of Eq. (41) is itself a function of the vertical height, this variation must be integrated laterally across the circular surface in order to compare the computations with the measured mean values.

\section{4}

\section{Conclusions}

An analytical model for the computation of dropwise condensation heat transfer on a vertical surface is presented, which expresses the heat transfer coefficient in terms of the thermal properties of the condensing fluid, the nucleation site density, drop size distribution, departure size, and surface subcooling. Since basic principles are used in the derivation of the model, a minimum number of experimental parameters are necessary for computing the heat

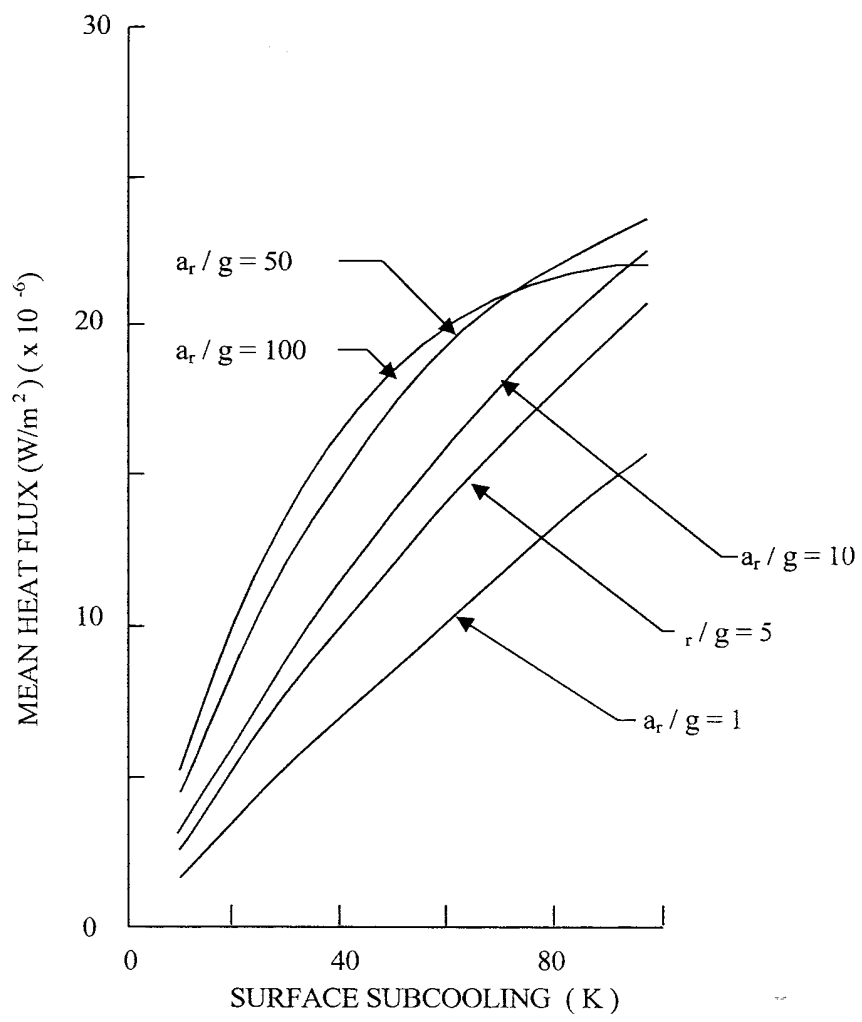

Fig. 11. Mean heat flux for a vertical plate of height $0.01 \mathrm{~m}$

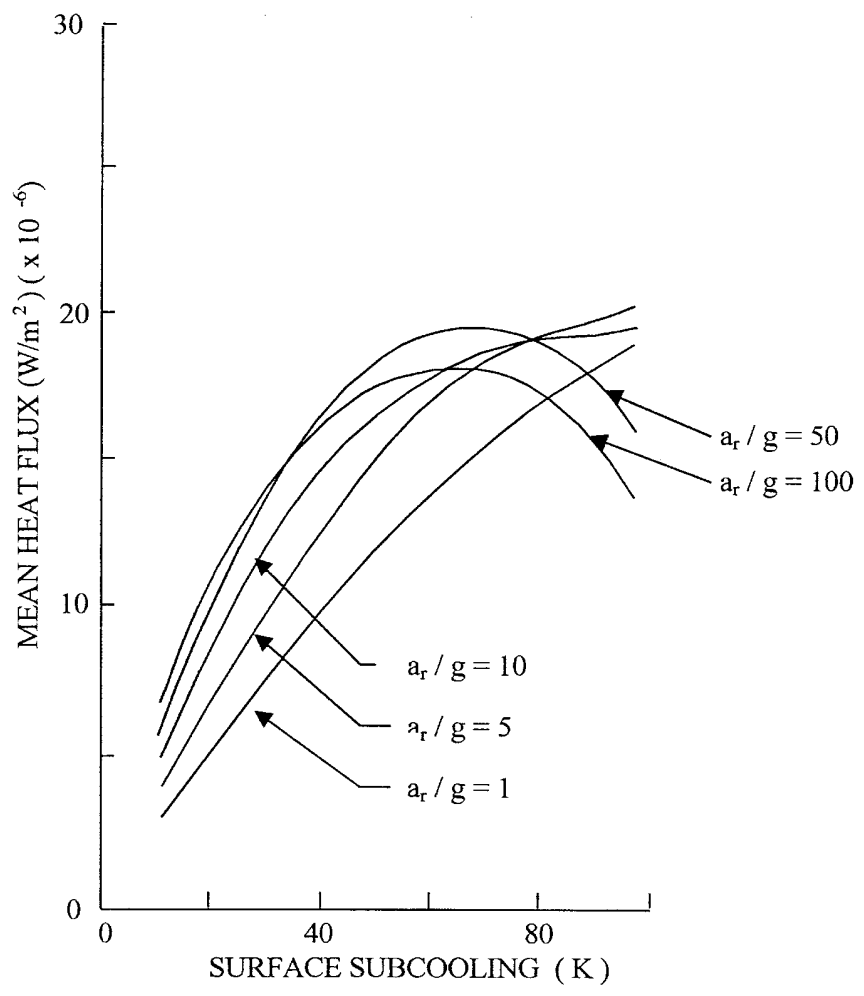

Fig. 12. Mean heat flux for a vertical plate of height $0.1 \mathrm{~m}$

transfer coefficient. These experimental parameters are: a coefficient $n$ used in the dropsize distribution, the velocity of the falling droplets and the distance between the nucleation sites $\left(2 r_{\mathrm{co}}\right)$. When compared with previous works, the present model introduces the following new features: 


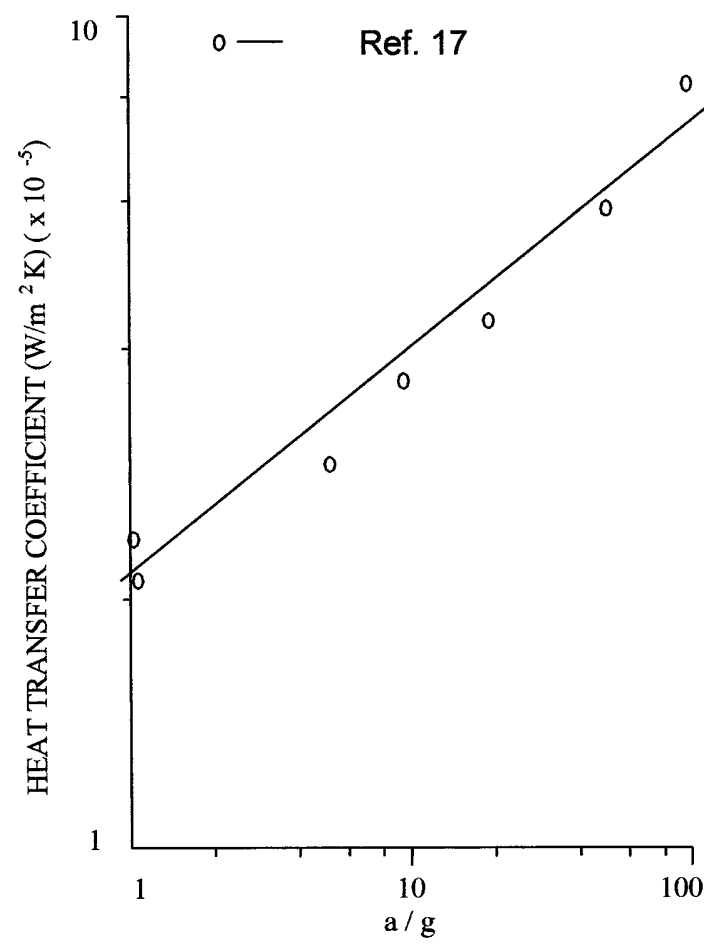

Fig. 13. Heat transfer coefficient versus nondimensional acceleration for a circular condensing surface

(i) It modifies the dropsize distribution at the drop sizes for which drop growth takes place by condensation on the droplets only.

(ii) It introduces the effect of the sweeping by departing droplets by carefully separating the sweeping mechanism from the other events taking place during dropwise condensation.

(iii) The blanketing or insulating effect of the falling droplets is introduced into the model.

The results of calculations clearly show that the heat flux is not a linear function of the surface subcooling (as is expected), but shows a "boiling curve type" behavior i.e. the heat transfer coefficient decreases at large surface subcoolings. The linear behavior occurs only at small surface subcoolings.

A major contribution of the model here is the demonstration of the existence of a maximum heat flux in dropwise condensation. The characteristics of the so-called boiling curve, the relationship between heat flux and the heater surface superheat, have been studied extensively. Similar experiments for condensation have been carried out, producing a corresponding heat flux versus temperature curve at gravitational acceleration, by Takeyama and Shimizu [19]. The experiments clearly showed the existence of a maximum heat flux for condensation. This may be considered to be an expected result, since the physical processes which govern the boiling and condensation phenomena have similarities. That an analogy exists between them is therefore not surprising. Although the experimental results cited above clearly show the existence of a maximum heat flux for condensation, none of the analytical models available to this point have predicted such a maximum heat flux due to the inadequate introduction of the sweeping process into the models.

Calculations conducted with the model developed here demonstrate that a plate of sufficient height (greater than $10 \mathrm{~cm}$ for water) will have a maximum heat flux with dropwise condensation, with variable condenser surface subcoolings, only if the body force ratio $a_{r} / g$ is greater than 5 . At body force ratios less than this the blanketing or insulating effect will not be sufficient to provide a maximum in the heat flux as surface subcooling increases.

\section{References}

1. Schmidt E; Schuring W; Sellschopp W (1930) Tech Mech Thermodyn 1: 53

2. Lefevre EJ; Rose J (1966) A theory of heat transfer by dropwise condensation. 3rd Int Heat Transfer Conf (ASME-AIChE), vol. II, pp. 362-375

3. Sugawara S; Michiyoshi I (1956) Dropwise condensation. Memoirs of Faculty of Engineering Kyoto University 18: 84-96

4. Merte H; Yamali C; Son S (1986) A simple model for dropwise condensation heat transfer neglecting sweeping. Proc 8th Int Heat-Transfer Conf, San-Francisco, California 4: 1659-1664

5. Fatica N; Katz DL (1949) Dropwise condensation. Chem Eng Progress 45: 661-674

6. Shea FL; Krase NW (1940) Trans Am Inst Chem Engrs 36: 463-470

7. McCormick JL; Westwater JW (1965) Nucleation sites for dropwise condensation. Chem Eng Sci 20: 1021-1031

8. Peterson AC; Westwater JW (1965) Dropwise condensation of ethylene glycol. Presented at the 8th National Heat Transfer Conference (A.I.Ch.E-A.S.M.E.) Los Angeles, California

9. Umur A; Griffith P (1965) Mechanism of dropwise condensation. Trans ASME, J Heat Transfer 87C: 275-282

10. Ivanovskii MN; Subbotion VL; Milovanov YV (1967) Heat transfer with dropwise condensation of mercury vapour. Teploenergetika 14: 81-90

11. Hurst CJ; Olson DR (1973) Conduction through droplets during dropwise condensation. J Heat Transfer 95: 12-20

12. Graham C; Griffith P (1973) Drop size distributions and heat transfer in dropwise condensation. Int J Heat Mass Transfer 16: $337-349$

13. Krischer S; Grigull U (1971) Microscopic study of dropwise condensation. Warme- und Stoffübertragung 4: 48-59

14. Yamali C; Merte H (1984) Conduction heat transfer through condensation drops with variable interphase coefficients. Symposium on Fundamentals of Two-Phase Flow, Boiling and Condensation, ASME W/A Meeting 9-14 December, New Orleans, LA

15. Rose JW; Glicksman LR (1973) Dropwise condensation. The distribution of drop sizes. Int J Heat Mass Transfer 16: 411-425

16. Merte H; Yamali C (1983) Profile and departure size of condensation drops or vertical surfaces. Warme und Stoffübertragung 17: 171-180

17. Yamali C; Merte H (1999) Influence of sweeping on dropwise condensation with varying body force and surface subcooling. Int J Heat Mass Transfer 42: 2943-2953

18. Kawai T; Kamo N (1966) Study on application of dropwise condensation to steam condensers. Bulletin of JSME 9: 756767

19. Takeyama T; Schimizu S (1974) On the transition of dropwise-film condensation. Proc Fifth Int Heat Transfer Conf 3: 274-290

20. Yamali C (1983) Dropwise Condensation under High Gravity and at Large Subcooling. PhD Thesis, The University of Michigan, Ann Arbor, MI 